Research Paper

\title{
Characterization and potential roles of bone marrow-derived stromal cells in cancer development and metastasis
}

\author{
Hotaka Kawai ${ }^{1}$, Hidetsugu Tsujigiwa², Chong Huat Siar³, Keisuke Nakano${ }^{1}$, Kiyofumi Takabatake ${ }^{1}$, \\ Masae Fujii' ${ }^{1}$, Mei Hamada ${ }^{1}$, Ryo Tamamura ${ }^{4}$, Hitoshi Nagatsuka ${ }^{1}$
}

1. Department of Oral Pathology and Medicine, Graduate School of Medicine, Dentistry and Pharmaceutical Sciences, Okayama University, Okayama, Japan

2. Department of Life Science, Faculty of Science, Okayama University of Science, Okayama, Japan

3. Department of Oral and Maxillofacial Clinical Sciences, Faculty of Dentistry, University of Malaya, Kuala Lumpur, Malaysia

4. Department of Histology, Nihon University School of Dentistry at Matsudo, Japan

$\square$ Corresponding author: Hotaka Kawai, Department of Oral Pathology and Medicine, Graduate School of Medicine, Dentistry and Pharmaceutical Sciences, Okayama University, 2-5-1 Shikata-Cho, Okayama, 700-8558 Japan; Tel: +81 86235 6651; Fax: +81 86235 6654; E-mail: de18018@s.okayama-u.ac.jp

(c) Ivyspring International Publisher. This is an open access article distributed under the terms of the Creative Commons Attribution (CC BY-NC) license (https://creativecommons.org/licenses/by-nc/4.0/). See http://ivyspring.com/terms for full terms and conditions.

Received: 2017.12.14; Accepted: 2018.04.27; Published: 2018.09.07

\begin{abstract}
Background: The tumor microenvironment and its stromal cells play an important role in cancer development and metastasis. Bone marrow-derived cells (BMDCs), a rich source of hematopoietic and mesenchymal stem cells, putatively contribute to this tumoral stroma. However their characteristics and roles within the tumor microenvironment are unclear. In the present study, BMDCs in the tumor microenvironment were traced using the green fluorescent protein (GFP) bone marrow transplantation model.

Methods: C57BL/6 mice were irradiated and rescued by bone marrow transplantation from GFP-transgenic mice. Lewis lung cancer cells were inoculated into the mice to generate subcutaneous allograft tumors or lung metastases. Confocal microscopy, immunohistochemistry for GFP, $\alpha$-SMA, CD11b, CD31, CD34 and CD105, and double-fluorescent immunohistochemistry for GFP-CD11b, GFP-CD105 and GFP-CD31 were performed.

Results: Round and dendritic-shaped GFP-positive mononuclear cells constituted a significant stromal subpopulation in primary tumor peripheral area (PA) and metastatic tumor area (MA) microenvironment, thus implicating an invasive and metastatic role for these cells. CD $1 \mathrm{lb}$ co-expression in GFP-positive cells suggests that round/dendritic cell subpopulations are possibly BM-derived macrophages. Identification of GFP-positive mononuclear infiltrates co-expressing CD31 suggests that these cells might be BM-derived angioblasts, whereas their non-reactivity for CD34, CD105 and $\alpha$-SMA implies an altered vascular phenotype distinct from endothelial cells. Significant upregulation of GFP-positive, CD31-positive and GFP/CD31 double-positive cell densities positively correlated with PA and MA $(P<0.05)$.

Conclusion: Taken together, in vivo evidence of traceable GFP-positive BMDCs in primary and metastatic tumor microenvironment suggests that recruited BMDCs might partake in cancer invasion and metastasis, possess multilineage potency and promote angiogenesis.
\end{abstract}

Key words: cancer microenvironment, bone marrow derived cells, lung cancer, animal model

\section{Introduction}

The tumor microenvironment plays a pivotal role in cancer development and progression [1]. Unlike its naive counterpart which maintains physiological homeostasis of normal tissues, the tumor microenvironment is a pathologically active niche that contributes to tumor growth and metastasis by collaborating with tumor cells $[2,3]$. Within this tumoral stroma, many structurally and functionally 
essential stromal elements including cancerassociated fibroblasts (CAFs) and myofibroblasts participate in the regulation of solid tumor progression [4-7]. In addition other tumor-associated stromal cells namely macrophages, endothelial cells and $\mathrm{T}$ cells contribute to tumor invasion and metastasis [8-10].

Bone marrow is a complex tissue comprising a heterogeneous population of multipotent cells including hematopoietic and mesenchymal stem cells. These bone marrow-derived cells (BMDCs) have been frequently investigated as a cell source for regenerative medicine application [11]. In cancers, thus far it is known that circulating BMDCs switched their phenotypes to hepatocytes [11], endothelial cells [12,13], myofibroblasts [14] and gastrointestinal epithelial cells in vivo [15-17]. Furthermore, in gastric cancer, the stromal cells including CAFs were reported to originate from BMDC [18].

Although the multilineage differentiation capacity of BMDC has been explored [11-18], the distribution, differentiation characteristics and roles of BMDCs in cancer development and metastasis remain unclear. To investigate how BMDCs influence tumor invasion and metastasis, a mouse bone marrow transplantation model was created by grafting bone marrow from green fluorescent protein (GFP) mice into isogenic wild-type mice. These mice were transplanted with Lewis lung cancer (LLC) cells injected subcutaneously into their back or tail vein. This mouse model allowed tracking of BMDC by tracing for GFP-positive cells. The location of GFP-positive cells in tumor tissues, and GFP-positive cell differentiation were also determined.

\section{Material and Methods}

\section{Experimental animals}

A total of 23 female mice was used. These consisted of eight GFP transgenic mice (C57BL/6-Tg [CAG- EGFP] OsbC14-Y01-FM131) (gift from Professor Masaru Okabe, Genome Information Research Center, Osaka University, Suita, Japan) and 15 C57BL/6 mice purchased from Okayama University Animal Center.

All experimental protocols complied with the guidelines of the Okayama University Care and Use of Laboratory Animals. This research was approved by the Animal Experiment Control Committee of the Okayama University Graduate School of Medicine, Dentistry and Pharmaceutical Sciences (05-006-099).

\section{Bone marrow transplantation}

Bone marrow transplantation was carried out according to a standard protocol as described previously [19]. Bone marrow cells from GFP mice were collected by introducing DMEM (Invitrogen, Grand Island, NY) into the marrow space. Cells were resuspended with HBSS into approximately $1.0 \times 10^{7}$ cells/0.2 ml. Meanwhile, 8-week old female C57BL/ 6 recipient mice were subjected to $10 \mathrm{~Gy}$ of lethal whole-body irradiation, and resuspended bone marrow cells were injected into the tail vein of recipient mice. The bone marrow was examined by GFP immunohistochemistry (IHC) 4 weeks after transplantation.

\section{Tumor cell culture}

LLC cells purchased from American Type Culture Collection (ATCC Manassas,VA, USA) were maintained in DMEM supplemented with $10 \%$ FBS, $100 \mathrm{U} / \mathrm{ml}$ antimyotic-antibiotic $100 \mathrm{mg} / \mathrm{ml}$ at $37^{\circ} \mathrm{C}$ in a humidified atmosphere at $5 \% \mathrm{CO}_{2}$.

\section{Tumor transplantation}

12-week-old C57BL/6J female mice that received GFP bone marrow transplantation were injected subcutaneously into the back with $1.0 \times 10^{6}$ LLC cells $(n=5)$ or injected into the tail vein with $5.0 \times 10^{5}$ LLC cells $(n=5)$. We defined the former as primary model, and the latter as metastatic model. The bone marrow transplantation mouse was defined as control. At 28 days, all mice were sacrificed and the specimens were harvested for analysis.

\section{Histological examination}

All excised tumor tissues were fixed in $4 \%$ paraformaldehyde for $12 \mathrm{~h}$. Tissues were processed routinely, embedded in paraffin wax, and 5 micron-thick serial sections were prepared. These sections were used for hematoxylin-eosin (HE) staining, IHC and double fluorescent IHC.

\section{Immunohistochemistry}

IHC was carried out using the antibodies detailed in Table 1. Following antigen retrieval, sections were treated with $10 \%$ normal serum for 30 min, and then doing incubation with primary antibodies at $4^{\circ} \mathrm{C}$ overnight. The immunoreactive site was identified using the avidin-biotin complex method (Vector Lab, Burlingame, CA, USA).

\section{Double-fluorescent IHC staining}

Double-fluorescent IHC for GFP-CD11b, GFP-CD105, and GFP-CD31 was performed using GFP monoclonal antibody (Abcam, Tokyo, Japan). The secondary antibodies used are detailed in Table 2. Antibodies were diluted with Can Get SignalÒ (TOYOBO, Osaka, Japan). After antigen retrieval, sections were treated with Block AceO (DS Pharma Bio-medical, Osaka, Japan) for $30 \mathrm{~min}$ at room temperature. Specimens were incubated with primary 
antibodies at $4{ }^{\circ} \mathrm{C}$ overnight. These specimens were then incubated with secondary antibody at a dilution of 1:200 for $1 \mathrm{~h}$ at room temperature. After the reaction, the specimens were stained with $1 \mathrm{lg} / \mathrm{ml}$ of DAPI (Dojindo Laboratories, Kumamoto, Japan).

\section{Cell counting}

Cell counting was performed according to three areas (Fig. 1A) of division: 1) peripheral area (PA) (Fig. 1C) of primary tumor model (tumoral tissue interacted with peripheral stroma); 2) central area (CA) (Fig. 1D) of primary tumor model (tumoral tissue away from peripheral stroma); and 3) metastatic area (MA) (Fig. 1E) of metastatic tumor model (tumoral tissue metastasized in lung). The subsequent histological analysis and characterization of LLC cells and BMDC were made based on this area division.
After counterstaining with hematoxylin, sections were examined under a microscope at 400 magnification. Ten areas were randomly chosen in each sample. The number of positively labelled cells was counted manually, the average was obtained and compared among the 3 groups.

\section{Vessel density}

IHC was performed using antibody against mouse CD105, and the density of tumor vessels was scored by BZ-X700 all in one microscope (Keyence, Osaka, Japan).

\section{Statistical analyses}

The statistical data was presented as the mean \pm SEM. The comparisons between the mean variables of the two groups were performed using Student's $t$ test. The difference was considered significant at $\mathrm{P}<0.05$.

Table 1. Antibodies used in immunohistochemistry

\begin{tabular}{|c|c|c|c|c|}
\hline Primary antibody & Immunized animal & Antigen retrival & Dilution & Supplier \\
\hline \multirow[t]{2}{*}{ GFP } & Rabbit & $\begin{array}{l}\text { Heated in } 0.01 \mathrm{~mol} / \mathrm{L} \text { citrate } \\
\text { buffer for } 1 \mathrm{~min}\end{array}$ & $1: 200$ & MBL \\
\hline & Goat & $\begin{array}{l}\text { Heated in } 0.01 \mathrm{~mol} / \mathrm{L} \text { citrate } \\
\text { buffer for } 1 \mathrm{~min}\end{array}$ & $1: 200$ & abcam \\
\hline $\mathrm{CD} 11 \mathrm{~b}$ & Rabbit & $\begin{array}{l}\text { Heated in } 0.01 \mathrm{~mol} / \mathrm{L} \text { citrate } \\
\text { buffer for } 1 \mathrm{~min}\end{array}$ & $1: 1000$ & abcam \\
\hline$\alpha$-SMA & Rabbit & $0.1 \%$ Trypsin at $37{ }^{\circ} \mathrm{C}, 5 \mathrm{~min}$ & $1: 200$ & abcam \\
\hline CD34 & Rabbit & $0.1 \%$ Trypsin at $37{ }^{\circ} \mathrm{C}, 5 \mathrm{~min}$ & $1: 200$ & abcam \\
\hline CD31 & Rat & $0.1 \%$ Trypsin at $37{ }^{\circ} \mathrm{C}, 5 \mathrm{~min}$ & $1: 1000$ & abcam \\
\hline $\mathrm{CD} 105$ & Goat & $0.1 \%$ Trypsin at $37{ }^{\circ} \mathrm{C}, 5 \mathrm{~min}$ & $1: 50$ & $\mathrm{R} \& \mathrm{D}$ \\
\hline $\mathrm{CD} 3$ & Rabbit & $\begin{array}{l}\text { Heated in } 0.01 \mathrm{~mol} / \mathrm{L} \text { citrate } \\
\text { buffer for } 1 \mathrm{~min}\end{array}$ & $1: 200$ & abcam \\
\hline
\end{tabular}

Table 2. Antibodies used in double-fluorescent immunohistochemistry

\begin{tabular}{cccc}
\hline Second antibody & Immunized animal & Fluorescent dye & Supplier \\
\hline \hline Anti Goat IgG & Donkey & Alexa Flour 568 & Thermo \\
& Donkey & Alexa Flour 488 & Thermo \\
Anti Rat IgG & Donkey & Alexa Flour 488 & Thermo \\
Anti Rabbit IgG & Donkey & Alexa Flour 568 & Thermo \\
& & & Alexa Flour 488
\end{tabular}




\section{Results}

\section{Morphological characterization of primary and metastatic models}

Solid LLC tumors grew in both primary and metastatic models (Fig. 1A, 1B). The primary tumors consisted of a peripheral area of loosely arranged spindle-shaped tumor cells which were in contact with stromal cells (Fig. 1C). In the central tumor area, these tumor cells formed a compact pleomorphic population of large, round-to-polygonal cells with nuclear atypia. These cells were not in contact with stromal cells (Fig. 1D).

In the metastatic model, the tumors presented as multifocal nodular masses (Fig. 1B). Each metastatic nodule consisted of a central area of compact round-to-polygonal tumor cells (not shown), and peripheral loosely-arranged spindle-shaped cells, the latter of which were in close contact with the stromal cellular components (Fig. 1E).

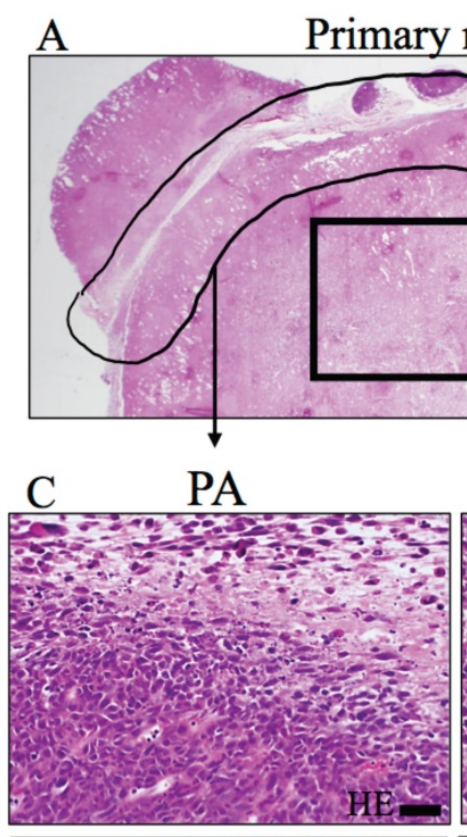

\section{B Metastatic model}
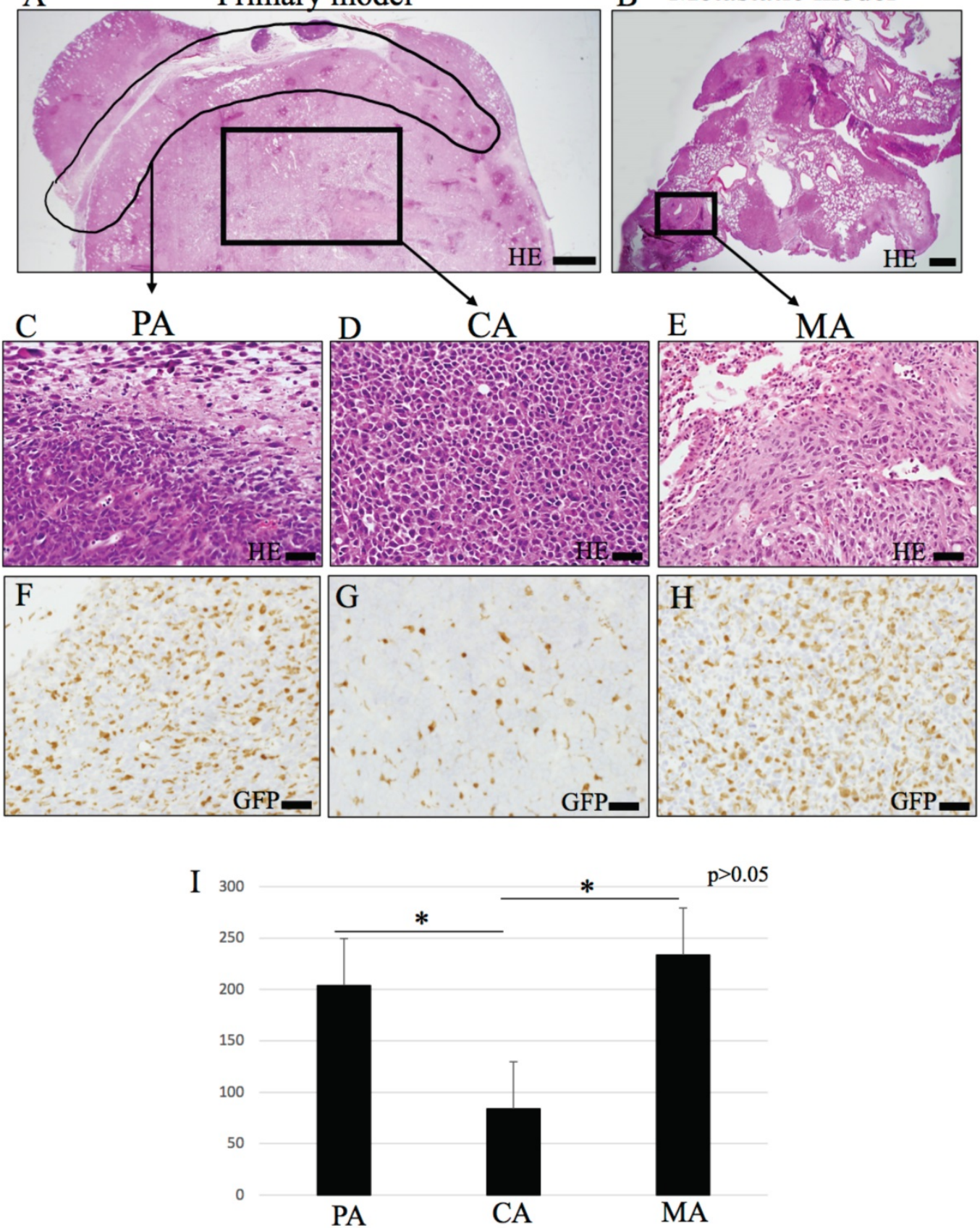

Figure 1. Histopathological appearances and IHC of GFP. (A-E) Hematoxylin-eosin (HE) staining. Loupe image of (A) primary model and (B) metastasis model. (C) peripheral area (PA). Spindle tumor cells are observed and infiltrate to peripheral tissue. (D) central area (CA). Round or angulated tumor cells are observed. (E) metastasis area (MA). (F-H) GFP immunohistochemical staining (IHC). GFP positive cells are mononuclear cells and round or dendritic cells. (F) PA, (G) CA, (H) MA. (I) The number of GFP positive cells are significantly higher in PA and MA than CA $(P<0.05)$. In MA, GFP positive cells infiltrate tumor diffuse, and cell density is similar PA. Bars: AB, $500 \mu \mathrm{m}$; $\mathrm{C}-\mathrm{H}, 20$ $\mu \mathrm{m}$. 

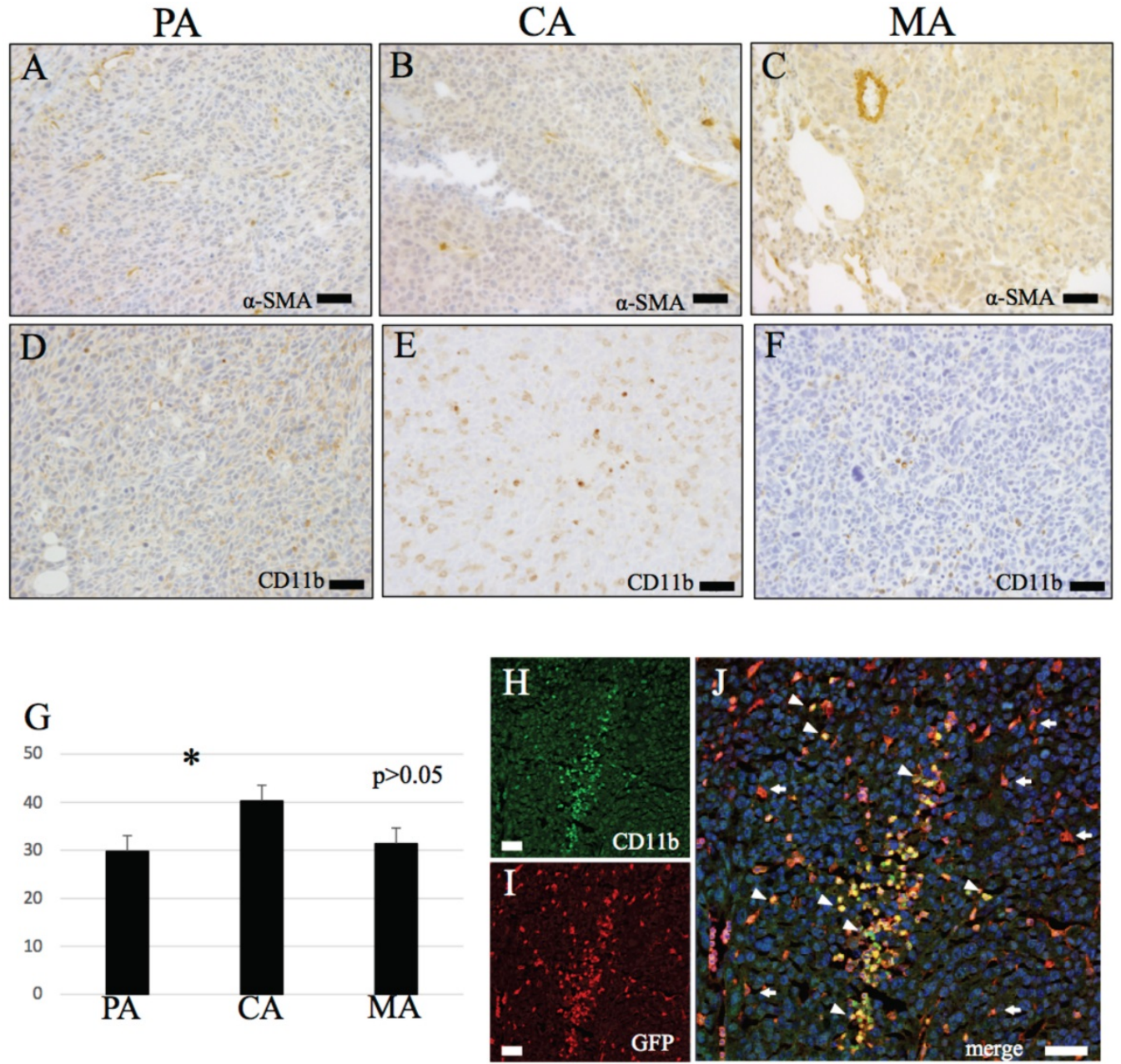

Figure 2. IHC features of $\alpha-S M A$ and CDI lb. (A-C) a-SMA. (A) PA, (B) CA, (C) MA. (D-F) CDI lb. Round shape CDI lb positive cells observed. (D) PA, (E) CA, (F) MA. (G) The number of CD $1 \mathrm{lb}$ positive cells are significantly higher in PA than CA ( $\mathrm{P}<0.05)$. (H-J) Immunofluorescence double stain with CDI1b and GFP. (H) CDI Ib, (I) GFP, (J) merge image of CDI lb and GFP. Bars: A-F and H-J, $20 \mu \mathrm{m}$.

\section{IHC localization of GFP-positive cells}

Abundant GFP-positive cells infiltrated the tumor microenvironment of both models. These GFP-positive cells presented as round or dendritic mononuclear cells (Fig. 1F-H).

\section{Expression of various markers by tumor stromal cells and comparison with GFP. positive cells}

a-SMA labelled smooth muscle of stromal blood vessels in both primary and metastatic models. No difference in a-SMA distribution in CA, PA and MA was observed. GFP-positive round or dendritic cells were negative for a-SMA (Fig. 2A-C). A small number of spindle-shaped cells that did not form a-SMA positive lumen was observed in PA (Fig. 2A).

Abundant CD11b-positive cells infiltrated the tumoral stroma of both primary and metastatic models. These cells were spherical or dendritic in shape, morphologically similar to GFP-positive cells (Fig. 2D-F). Numerous of these spherical
CD11b-positive cells were also observed in necrotic tumor areas in CA.

CD31, CD34 and CD105 were positive for vascular endothelial cells of blood vessels in the tumor tissue (Figs. 3A-F, 4A-C). These vessels have central lumens or slit-like spaces. In addition, most of these vessels in PA and MA have smaller lumens whereas numerous mature blood vessels with large lumens were observed in CA (Figs. 3A-F, 4A-C). Spherical or dendritic stromal cells were positive for CD31 but were nonreactive for CD34 and CD105 (Fig. 4A-C). These cells morphologically resembled GFP-positive cells.

\section{Analysis of double-fluorescent IHC staining}

CD $11 \mathrm{~b}$ and GFP

Spherical GFP-positive cells co-expressing CD11b were observed in both tumor models. These double positive cells infiltrated necrotic tumor sites of CA (Fig. 2H-J) and to a lesser extent those of PA and MA. 


\section{105 and GFP}

In all sites examined for both primary tumor and metastatic lesion, double positive CD105 and GFP endothelial cells were not observed (Fig. 3G-I).

\section{CD31 and CD 105}

Both CD31 and CD105 were positive in vascular endothelial cells showing lumen formation (Fig. 4G arrow). In addition CD31 was positive in spherical or dendritic cells with no evidence of lumen formation (Fig. 4G arrowhead).

\section{CD31 and GFP}

In PA and MA, spherical cells double positive for CD31 and GFP were observed (Fig. 4H-J).

\section{Cell counting analysis of IHC positive cells}

Results of cell-counting based on IHC staining for GFP, CD11b, CD31 and double fluorescent IHC for GFP-CD31 according to the three area divisions PA, CA and MA are shown in the histograms (Figs. 1I, 2G, $4 \mathrm{D}$ and $4 \mathrm{~K})$. PA and MA consistently showed significantly higher scores for GFP-positive cells (Fig. 1I), CD31-positive cells (Fig. 4D) and CD31/GFP double positive cells than CA (Fig. $4 \mathrm{~K})(P<0.05)$. Only CD11b-positive cells scored highest in CA.

\section{Measurement of blood vessel area}

Data based on measurement of blood vessel area in CA, PA and MA are shown in Fig 3J. Both PA and MA showed significantly higher blood vessel area than CA. (Fig. 3J).
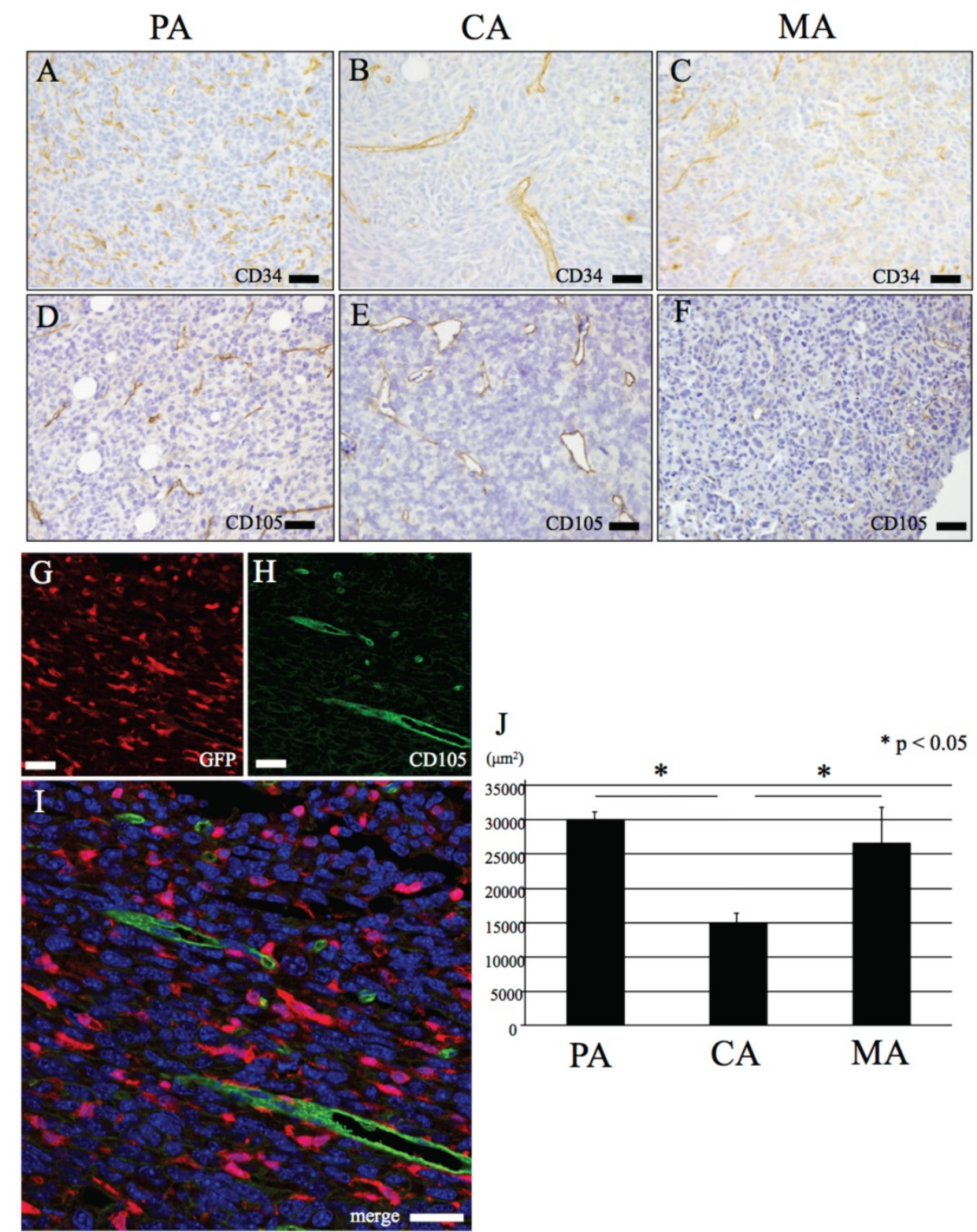

Figure 3. IHC features of CD34 and CD105. (A-C) CD34. (A) PA, (B) CA, (C) MA. (D-F) CD105. (D) PA, (E) CA, (F) MA. Positive staining of CD34 and CD105 shown in only vascular endothelial cells. (G-H) Immunofluorescence double stain with GFP and CD105. (G) GFP, (H) CD105, (C) merge image of CD105 and GFP. Endothelial cells (green) are negative for GFP (red). () PA and MA vascular density is significant higher than CA ( $<<0.05)$. Bars: A-I, $20 \mu \mathrm{m}$. 

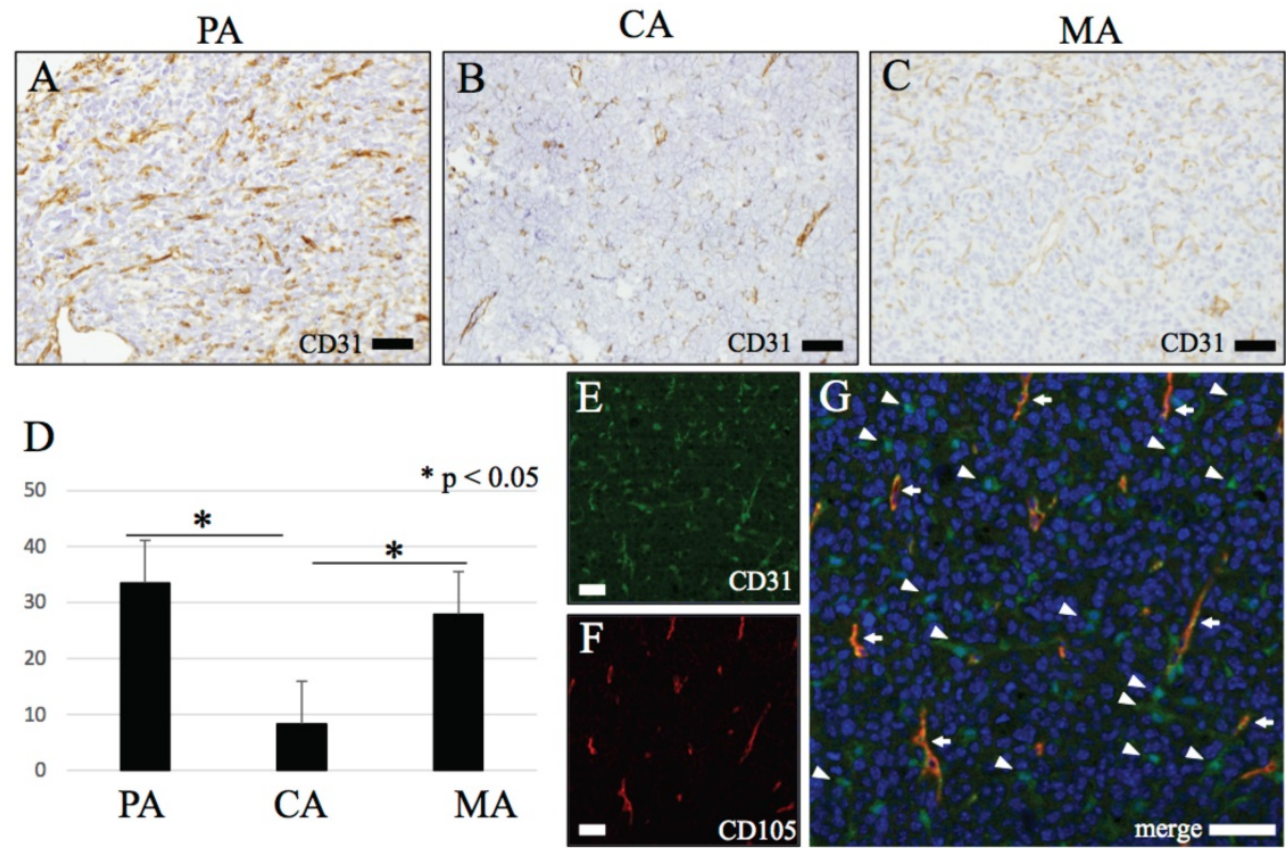

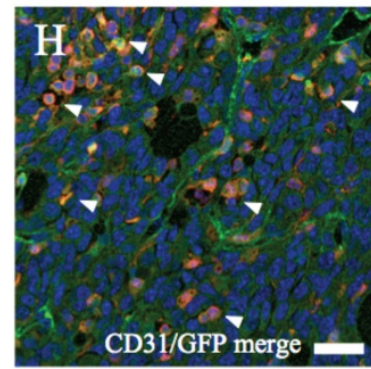

PA

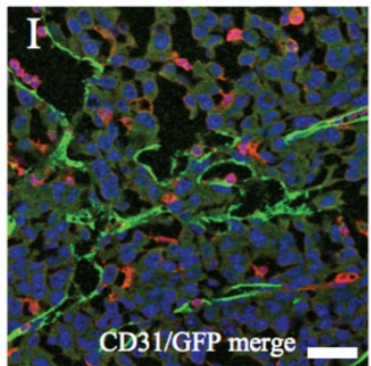

CA

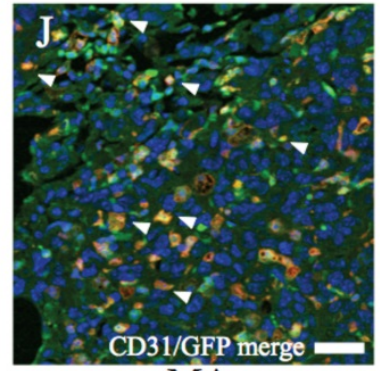

MA

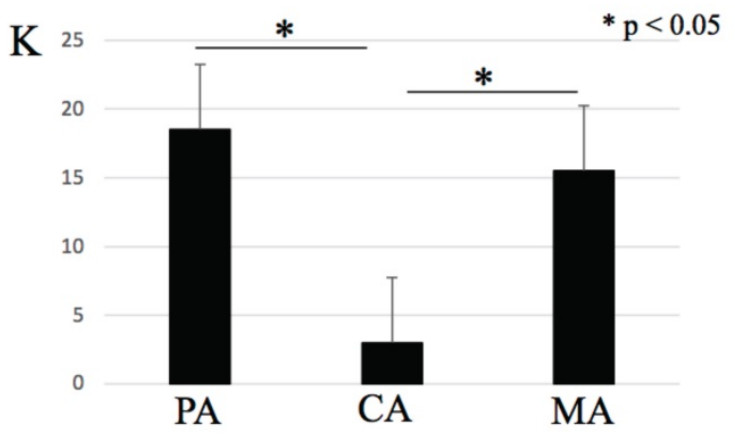

Figure 4. IHC features of CD31. (A-C) CD31. (A) PA, (B) CA, (C) MA. (D) The number of CD31 positive round or dendritic cells are significantly higher in PA and MA than $C A(p<0.05)$. (H-J) Immunofluorescence double stain with CD31 and GFP. (H-J) merge image of CD31 and GFP. Double positive mononuclear cells are mainly observed in PA and MA (arrow head), but few numbers in CA. (K) The number of CD31/GFP double positive cells are significantly higher in PA and MA than CA ( $P<0.05)$. Bars: A-C and E-J, $20 \mu \mathrm{m}$.

\section{Discussion}

The heterogeneity of the stromal cellular populations within the tumor microenvironment makes definitive characterization inherently challenging $[3,4,6]$. Tracing BMDCs and their differentiated phenotypes in the neoplastic cell extrinsic environment formed our main research goal. The GFP bone marrow transplantation model offers an attractive approach because it enables the tracking of BMDCs in both their engraftment and homing sites
$[18,19]$. By using this bone marrow transplantation model harboring GFP-labeled BMDCs, we successfully illustrated here the distribution of the various cellular constituents derived from the transplanted BMDCs within the primary and metastatic tumor microenvironment. We also provided morphologic evidence of multilineage differentiation by these cells in both tumor sites. Their characteristics and potential roles are discussed further below. 
Current data based on GFP positivity suggest that a significant population of stromal cells in the primary and metastatic tumor microenvironment is BM-derived. Abundant GFP-labelled BMDCs have been previously described in gastric and colon cancer stroma $[18,20]$. In this study, an invasive role for BMDC was demonstrated with evident upregulation of GFP-positive cellular infiltrates concentrated in the peripheral locations of the primary tumor model. Furthermore the close proximity between these GFP-positive cellular infiltrates and spindle-shaped tumor cells along the peripheral/invasive front was interpreted to represent tumor-stromal cell interactions. However actual fusion between these cell types (multiploidal cells) was not observed [21]. Furthermore, a metastatic role for BMDC was demonstrated here based on the enhanced presence of GFP-positive cellular infiltrates in the lung metastatic tumor nodules. Liver metastases of colon cancer were also known to show abundant GFP-positive cells [20].

Current data further revealed that GFP positivity in the primary and metastatic tumoral stroma was predominantly monopolized by mononuclear infiltrates with round/spherical and dendritic cell morphology, thus confirming that these cells are BM-derived. Co-expression of CD11b by these GFP-positive cells suggests that these round/spherical and dendritic cell subpopulations are most likely BM-derived mononuclear phagocyte/macrophages. CD11b is a known marker for monocytes/macrophages and microglia [22]. Therefore CD11b/GFP double-positive cells that accumulated in the tumor necrotic sites of CA were thought to be macrophages that function to phagocytize necrotic tissues. On the other hand, CD11b-positive cells elsewhere in the tumoral stroma were suggested to represent tumor-associated macrophages (TAM). TAM is known to be involved in tumor growth and metastasis [23], thus suggesting that CD11b-positive BMDCs found in tumor tissue margins and metastatic lesions may be related to TAMs. Recent evidence indicates that BM-derived macrophages have an angiogenesis promoting action in tumor angiogenesis [24,25]. Therefore, the observed significantly higher CD11b-positive BMDCs densities within ischemic/necrotic central tumor areas suggest that macrophages function to phagocytize foreign matter while TAMs are involved in angiogenesis.

Tumor vessels play a pivotal role in tumor development. To clarify the relative contribution of BMDCs in the tumoral vasculature of primary and metastatic tumor microenvironment, the angiogenic index based on IHC using CD31, CD34 and CD105, and dual fluorescent IHC using GFP-CD31 and GFP-CD105 in both primary and metastatic sites was estimated. Current results revealed that endothelial cells lining blood vessels in primary and metastatic tumoral stroma were not doubly positive for GFP and CD105, thus dismissing that these blood vessels are BM-derived. Instead GFP-CD31 dual positivity was detected in spherical/dendritic cells, implying that these might be BM-derived circulating angioblasts. BM-derived GFP-positive and CD31-positive cells were however not detected in colon cancer allografts [20]. These consolidated findings indicate that tumor vessels originate mostly from host tissues rather than BM-derived circulating angioblasts. Recent evidence suggests that endothelial cells may originate from either endothelial progenitor cells (EPCs) in the bone marrow, angioblasts or vascular stem cells [26-2 8]. A favored view is that EPCs are BM-derived on grounds that blood cells and endothelial cells are generated from the same progenitor cell, and EPCs expressed the same antigens, such as CD34, CD31, and Tie-2 as hematopoietic precursor cells [29]. These data support the notion that not only the existing blood vessels but also EPCs in the BM might have been recruited during angiogenesis. Moreover, other data indicate that BMDCs might differentiate into endothelial cells during vascular repair in ischemic lesions including myocardial infarction. CD31 positive BMDC support angiogenesis strongly, but these cells didn't different to endothelial cells [30- 32]. Tumor-associated endothelial cells differ genetically from normal vascular endothelium [33]. Whether GPF/CD31-positive cells identified here belong to this subset of tumor-associated endothelial cells requires further investigations [34].

An earlier report described that GFP-positive cells frequently differentiated into vimentin-positive and $\alpha$-sma-positive myofibroblasts and interstitial cells that not only form a capsule but also infiltrated the colon cancer allografts [20]. Another study propounded that BMDCs might change to cancer-associated orthotopic myofibroblasts by the education of gastric cells [18]. In contrast to these reports, our results disclosed that a-SMA was expressed by vascular smooth muscle in the tumor tissues, and by spindle-shaped cells considered to be myofibroblasts in the peripheral portion of the tumor. However, neither of these cell types were GFP-positive, thus precluding that they are BM-derived stromal affiliates. Moreover, GFP-positive round and dendritic cells were negative for a-SMA. Based on these findings, we surmised that GFP-negative a-SMA-positive spindle cells might represent cancer-asssociated fibroblasts (CAFs) mobilized from the surrounding tumoral stroma rather than from the bone marrow $[5-7,14]$. 


\section{Conclusion}

In conclusion, present findings based on the GFP mouse bone marrow transplantation model, yielded in vivo evidence of BMDC involvement during cancer development and metastasis. Data on distinct distribution characteristics of GFP-positive BMDCs in primary and metastatic tumor microenvironment suggest that recruited BMDCs might be involved in cancer invasion and metastasis, while identification of GFP-positive macrophages, dendritic cells and circulating angioblasts attests to their multilineage potency and in turn the differential roles of these cells in tumor angiogenesis.

\section{Acknowledgements}

This study was jointly funded by the Japan Society for Promotion of Science (JSPS) KAKENHI Grant-in-Aid for Scientific Research (26462783) and (16K11441) and the Ministry of Health Malaysia Fundamental Research Grant FP032-2015A.

\section{Competing Interests}

The authors have declared that no competing interest exists.

\section{References}

1. Kessenbrock K, Plaks V, Werb Z. Matrix metalloproteinases: regulators of the tumor microenvironment. Cell. 2010; 141: 52-67.

2. Mantovani A, Allavena P, Sica A, et al. Cancer-related inflammation. Nature. 2008; 454: 436-444.

3. Whiteside TL. The tumor microenvironment and its role in promoting tumor growth. Oncogene. 2008; 27: 5904-5912.

4. Yashiro M, Hirakawa K. Cancer-stromal interactions in scirrhous gastric carcinoma. Cancer Microenviron. 2010; 3: 127-135.

5. Fuyuhiro Y, Yashiro M, Noda S, et al. Cancer-associated orthotopicmyofibroblasts stimulates the motility of gastric carcinoma cells. Cancer science. 2012; 103: 797-805

6. Tripathi M, Billet S, Bhowmick NA. Understanding the role of stromal fibroblasts in cancer progression. Cell AdhMigr. 2012; 6: 231-235.

7. Harper J, Sainson RC. Regulation of the anti-tumour immune response by cancer-associated fibroblasts. Semin Cancer Biol. 2014; 25: 69-77.

8. Damya L, Kiavah M, Eva VO, et al. Tumor-associated macrophages in breast cancer: distinct subsets, distinct functions. Int. J. Dev. Biol. 2011; 55: 861-867.

9. Piao Y, Park SY,Henry V, et al. Novel MET/TIE2/VEGFR2 inhibitor altiratinib inhibits tumor growth and invasiveness in bevacizumab-resistant glioblastoma mouse models. Neuro-Oncology. 2016; 18: 1230-1241.

10. Rothschild E, Banerjee D. A review on the breast cancer microenvironment and therapeutic opportunities. Breast Cancer (Auckl). 2015; 9: 7-15

11. Petersen BE, Bowen WC, Patrene KD, et al. Bone marrow as a potential source of hepatic oval cells. Science. 1999; 284: 1168-1170.

12. Aghi M, Chiocca EA. Contribution of bone marrow-derived cells to blood vessels in ischemic tissues and tumors. Mol. Ther, 2005; 12: 994-1005.

13. Lyden $\mathrm{D}$, Hattori $\mathrm{K}$, Dias $\mathrm{S}$, et al. Impaired recruitment of bonemarrow-derived endothelial and hematopoietic precursor cells blocks tumor angiogenesis and growth. Nat Med. 2001; 7: 1194-201.

14. Bamba S, Lee CY, Brittan M, et al. Bone marrow transplantation ameliorates pathology in interleukin-10 knockout colitic mice. Pathol. 2006; 209: 265-273.

15. Okamoto R, Yajima T, Yamazaki M, et al. Damaged epithelia regenerated by bone marrow-derived cells in the human gastrointestinal tract. Nat Med. 2002; 8: 1011-1017.

16. Komori M, Tsuji S, Tsujii M, et al. Efficiency of bone marrow-derived cells in regeneration of the stomach after induction of ethanol-induced ulcers in rats. J. Gastroenterol. 2001; 40: 591-599.

17. Komori M, Tsuji S, Tsujii M, et al. Involvement of bone marrow-derived cells in healing of experimental colitis in rats. Wound Repair Regen. 2005; 13: 109-118.

18. Kasashima $\mathrm{H}$, Yashiro $\mathrm{M}$, Nakamae $\mathrm{H}$, et al. Bone marrow-derived stromal cells are associated with gastric cancer progression. Br J Cancer. 2015; 113: $443-452$.
19. Hidetsugu T, Kazunori N, Takanori T, et al. The engraftment of transplanted bone marrow-derived cells into the olfactory epithelium. Brain Research 2005; 1052: 10-15.

20. Ishii S, Tsuji S. Involvement of bone marrow-derived stromal cells in gastrointestinal cancer development and metastasis. J Gastroenterolo. Hepatol. 2008; 2: 242-249.

21. Terada N, Hamazaki T, Oka M et al. Bone marrow cells adopt the phenotype of other cells by spontaneous cell fusion. Nature 2002; 416: 542-545.

22. Oscar R, Colegio NC, Alison LS, et al. Functional polarization of tumour-associated macrophages by tumour-derived lactic acid. Nature, 2013; $513,559-563$.

23. Komohara $Y$, Jinushi $M$, Takeya $M$. Clinical significance of macrophage heterogeneity in human malignant tumor. Cancer Sci. 2013; 105: 1-8.

24. Palma MD, Venneri MA, Roca C, et al. Targeting exogenous genes to tumor angiogenesis by transplantation of genetically modified hematopoietic stem cells. Nat. Med. 2003; 9: 789-795.

25. Ruhrberg C, Palma MD. A agent in cancer: deciphering macrophage roles in human tumors. Nat Med. 2010; 16: 861-862.

26. Boisset JC, Van CW, Andrieu-Soler C, et al. In vivo imaging of haematopoietic cells emerging from the mouse aortic endothelium. Nature. 2010; 464: 116-120.

27. Park C, Ma YD, Choi K. Evidence for the hemangioblast. Exp Hematol. 2005; 33: 965-970.

28. Wu SM, Chien KR, Mummery C. Origins and fates of cardiovascular progenitor cells. Cell. 2008; $132: 537-543$.

29. Asahara T, Murohara T, Sullivan A, et al. Isolation of putative progenitor endothelial cells for angiogenesis. Science. 1997; 275: 964-967.

30. Federica M, Marelli B, Marc C, et al. An immunologist's guide to CD31 function in T-cells. J. Cell Sci. 2013; 126: 2343-2352.

31. Can T, Feng-Sheng W, Kozaryn R, et al. Shock wave treatment induces angiogenesis and mobilizes endogenous CD31/CD34-positive endothelial cells in a hind limb ischemia model: Implications for angiogenesis and vasculogenesis. J. Thorac. Cardiovasc. Surg. 2013; 146: 971-978.

32. Kim SW, Kim H. Human peripheral blood-derived CD31+ cells have robust angiogenic and vasculogenic properties and are effective for treating ischemic vascular disease. J. Am. Coll. Cardiol. 2010; 56: 593-607.

33. Lee S, Yoon YS. Revisiting cardiovascular regeneration with bone marrow-derived angiogenic and vasculogenic cells. Bri. J. Pharmacol. 2013; $169: 290-303$.

34. Hida K, Hida Y, Amin DN, et al. Tumor associated endothelial cells with cytogenetic abnormalities. Cancer Res. 2004; 64: 8249-8255.

35. Kerbel, R.S. Tumor angiogenesis. N. Engl. J. Med. 2008; 358, 2039-2049.

36. Nolan DJ, Ciarrocchi A, Mellick AS, et al. Bone marrow-derived endothelial progenitor cells are a major determinant of nascent tumor neovascularization. Genes Dev. 2007; 21: 1546-1558.

37. Domenico R. The involvement of endothelial progenitor cells in tumor angiogenesis. J. Cell. Mol. Med.2004; 8: 294-300

38. Ishii, S., Tsuji, S. Involvement of bone marrow-derived stromal cells in gastrointestinal cancer development and metastasis. J Gastroenterolo. Hepatol. 2008; 2: 242-249.

39. Hyun-Jai C., Namho L. Role of host tissues for sustained humoral effects after endothelial progenitor cell transplantation into the ischemic heart. J. Exp. Med. 2007; 204: 3257-3269.

40. Sunyoung P, Christine M, Sorenson NS. PECAM-1 isoforms, eNOS and endoglin axis in regulation of angiogenesis. Clin. Sci. 2015; 129: 217-234. 